\title{
El viaje como barrera: diferencias y complejidades en el acceso a centros de salud en la ciudad de La Plata
}

María-Julieta López. Universidad Nacional de La Plata y Consejo Nacional de Investigaciones Científicas y Técnicas, La Plata, Argentina.

Laura-Cristina Aón. Universidad Nacional de La Plata y Consejo Nacional de Investigaciones Científicas y Técnicas, La Plata, Argentina.

María-Luciana Giglio. Universidad Nacional de La Plata y Consejo Nacional de Investigaciones Científicas y Técnicas, La Plata, Argentina.

Nadia Freaza. Universidad Nacional de La Plata y Consejo Nacional de Investigaciones Científicas y Técnicas, La Plata, Argentina.

Cristian Cola. Universidad Nacional de La Plata y Consejo Nacional de Investigaciones Científicas y Técnicas, La Plata, Argentina.

RESUMEN | Las estrategias que despliegan los distintos grupos sociales para moverse y acceder a las diferentes actividades en las ciudades son cada vez más complejas. En relación con la salud, la combinatoria entre las distancias vivienda-centros de salud y el nivel de ingreso de las personas y hogares, condiciona el acceso a este servicio e incide en la complejidad del viaje. Este artículo evidencia la criticidad de estas dos componentes a partir de un estudio de movilidad focalizado en los atractores de viaje de salud en la ciudad de La Plata. Esta estrategia metodológica permite caracterizar el viaje como lo hacen los estudios tradicionales, reconociendo los aspectos cuantitativos que caracterizan el viaje por motivos de salud, y también posibilita dimensionar el acceso a cada atractor visibilizando la necesidad de políticas públicas de movilidad como herramienta contra las desigualdades sociales en materia de salud.

PALABRAS CLAVE | desarrollo urbano, movilidad, planificación urbana.

ABSTRACT | The strategies deployed by social groups to move and access different activities in cities are becoming increasingly complex. In relation to health care, the spatial component and the income levels affect the access to this service and the complexity of the trip. This article evidences the complexity of these components from a study of mobility that focuses on the attractors of travel in the City of La Plata. This methodological strategy makes it possible to characterize the journey as traditional studies do, and it also makes it possible to measure access to each attractor, exposing the need for public mobility policies as a tool against social inequalities in health.

KEYWORDS | urban development, mobility, urban planning.

Recibido el 25 de septiembre de 2017, aprobado el 4 de marzo de 2018

E-mails: M.-J. López, arq.julietalopez@gmail.com | L.-C. Aón, laura.aon@gmail.com | M.-L. Giglio, giglio.luciana@hotmail.com | N.

Freaza, nadiafreaza@gmail.com | C. Cola, cristian.lp@live.com 


\section{El viaje por motivos de salud (a modo de introducción)}

La preeminencia del uso del automóvil y la carencia de planificación urbana integral son dos factores que explican la creciente complejidad de nuestros viajes urbanos. La promoción del uso de sistemas de transporte de pasajeros masivos (colectivos, tranvías, trenes y trolebuses) constituye, como política, la base de la sustentabilidad de una ciudad; sin embargo, en muchas ciudades argentinas, como en la ciudad de La Plata, el sistema público masivo de transporte no es promovido; carece de una planificación acorde a la actual cantidad de población urbana y periférica, a los actuales niveles de congestión de tránsito y a la forma expansiva en que la ciudad viene creciendo en las últimas décadas (Aón, Giglio \& Cola, 2017; Lozano, 2011; Ravella, 2000).

La falta de planificación se refleja en las deficiencias materiales del sistema de infraestructura de circulación y transporte, especialmente en las nuevas áreas residenciales periféricas y en las tradicionales áreas centrales (López, 2015). Es en este contexto que tanto los viajes regulares (por trabajo o estudio) como los eventuales (relacionados a la salud) se tornan en una experiencia dificultosa que impacta en los grupos con menor nivel de ingresos.

En el contexto de déficit de planificación urbana y preeminencia en el uso del auto, la distancia entre la vivienda y las actividades relacionadas con la salud crece y los distintos grupos de población viven cada vez más lejos de los equipamientos urbanos concentrados en áreas centrales, incrementando las distancias y diluyendo toda posible relación de proximidad con lo urbano. Por otro lado, la preeminencia en el uso del automóvil particular, la cual en general corresponde a sectores de población de ingresos medios y altos, ha provocado desde los últimos diez años una crítica congestión urbana que es causa estructural del deterioro del sistema público de transporte de pasajeros.

El viaje por salud ha sido considerado históricamente, en los estudios clásicos de transporte, como el motivo de viaje más relevante, después de los motivos regulares cotidianos, como trabajo y estudio (De la Barra, 1989; Olivera, Aón \& Ravella, 2003; Ortúzar \& Williamson, 1994); más recientemente, se lo ha comenzado a abordar desde diferentes estudios académicos de las ciencias sociales (Gutiérrez, 2009; Segura, 2012). Sin embargo, en Argentina, estos viajes en general no forman parte de la agenda de política pública.

En la ciudad de La Plata se han comenzado a impulsar, desde 2012, estudios específicos acerca de los usuarios de transporte público con destino a hospitales públicos de la ciudad, como iniciativa del poder ejecutivo municipal y, específicamente, de la dirección de transporte público de esos ańos. Dichos estudios permitieron dimensionar la demanda en este ámbito y dieron como resultado la implementación de ramales de transporte público específicos que conectan los centros de salud pública en la ciudad, apuntando a una solución del problema, pero sin resolverlo de manera estructural.

En Argentina, las políticas públicas son todavía insuficientes para dar respuesta a todas las dimensiones de la vida urbana; y, en particular, las políticas específicas relativas a mejorar o facilitar los viajes por salud han sido hasta ahora eventuales o 
marginales. Tanto las políticas urbanas dominadas por la lógica del mercado inmobiliario como la ausencia de políticas públicas integrales de movilidad por salud, producen, por defecto, exclusión social.

Es profusa la bibliografía reciente que aborda el estudio de la movilidad de sectores populares o poblaciones vulnerables, visibilizando las muy diversas situaciones desventajosas que condicionan las estrategias cotidianas de la población de menores ingresos para acceder a sus actividades y a los equipamientos urbanos (Bourdieu, 2000; Chaves, 2005, 2008; Figueroa, 2005, 2016; Gutiérrez, 2009; Jirón Martínez \& Fadda Cori, 1999; Jirón, Lange \& Bertrand, 2010; Landon, 2013; Ravella, 1994; Segura, 2006, 2012; Vignoli, 2008; Zucchini, 2015). Sin embargo, la literatura específica sobre la movilidad por motivos de salud es bastante escasa.

Como antecedente, existe un trabajo realizado para un sector del Área Metropolitana de Buenos Aires, donde se profundiza, desde la geografía cultural, el tema del viaje por salud de sectores populares. En él se pone en evidencia no solamente las dificultades y complejidad de grupos de embarazadas jóvenes pobres para acceder al sistema de salud y control del embarazo, sino, además, las contradicciones entre las políticas públicas de salud y transporte y los efectos de este desacople en las prácticas y satisfacción de los propósitos de esa movilidad por salud (Gutiérrez, 2009).

Asumimos que las políticas públicas en sistemas económicos capitalistas deben tender a corregir los desbalances del mercado y sus efectos sobre los sectores de población más vulnerables. Sin embargo, en nuestras ciudades, que van sumando progresivamente distancia entre las viviendas y las actividades urbanas, la política pública está en deuda. En primera instancia, por la falta de regulación del mercado de suelo y su consecuente expansión deteriorada, y también por falta de infraestructuras de servicios, circulación y transporte en las periferias urbanas pobres cuasi abandonadas. En segunda instancia, la política pública está en deuda con la movilidad y la salud por el riesgo de vida que conlleva la dificultad, demora o imposibilidad de acceder a un centro de salud.

En nuestro país están consolidados históricamente los sistemas de salud y educación públicos y gratuitos. En el caso del sistema de educación pública y gratuita, que alcanza al nivel universitario, esta política histórica es acompañada por políticas de gratuidad de movilidad que garantizan de manera completa el acceso a la educación. Sin embargo, esta mirada no se aplica en las políticas públicas de salud. Como señala Andrea Gutiérrez (2009), "la salud pública en Argentina es universal y gratuita, el acceso a la salud no" (s/p).

Dado que la movilidad y accesibilidad de los sectores populares es compleja y cada vez más crítica en la ciudad expandida y desde periferias monofuncionales (Abba, Kullock, Novick \& Schweitzer, 2011; Aón, Giglio \& Cola, 2017), es necesario construir conocimiento sobre el viaje por motivos de salud, con el fin de visibilizar las estrategias que despliegan las personas como argumentos posibles para futuras políticas públicas renovadas.

Particularmente en el contexto de desarrollo urbano y pobreza, se definen dos componentes clave y posibles sujetos de políticas públicas renovadas: por un lado, las relaciones espaciales vivienda-actividades de salud, mediadas por distancias crecientes y modos de transporte lentos, de difícil acceso material, alto costo e incertidumbre 
de funcionamiento o inserción urbana ${ }^{1}$ por otro lado, los niveles de ingreso de las familias y hogares, su estructura familiar, sus estrategias de supervivencia económica y las limitaciones o restricciones para resolver urgencias e importancias de salud entre los miembros de ese hogar. Las formas combinadas de estas componentes conforman auténticas maniobras para el acceso a la salud por parte de las familias y hogares. Jirón Martínez, Lange \& Bertrand (2010), investigadores de la movilidad y sectores vulnerables, señalan que las principales condicionantes al respecto son "las barreras financieras, físicas, organizacionales, temporales, de habilidades y tecnológicas” (pp. 44-45). El costo de los modos de transporte, la distancia viajada, las condiciones de seguridad y confort de los espacios de la movilidad, el despliegue de actividades del hogar para coordinar la vida cotidiana, el momento del viaje, las capacidades de los individuos para conducir (auto, moto, bicicleta) o para manejar tecnología que les permita facilitar el viaje o sustituirlo, todo con restricciones materiales y económicas, constituyen verdaderamente impedimentos o barreras.

En este marco, el trabajo que aquí se presenta busca dar cuenta de la problemática de movilidad en relación con la salud en la ciudad de La Plata, con énfasis en los efectos de las políticas públicas de salud, urbanas y de transporte en la población, según su nivel de ingreso y su localización residencial. Por otro lado, muestra la innovación metodológica en el estudio de movilidad al hacerlo a partir de la noción de "atractores de viaje", enfoque que supone construir información tomando un camino inverso al tradicional: realizando encuestas y relevamientos en las actividades urbanas más importantes de los hogares, dimensionando el itinerario origendestino y producción atracción del viaje, en relación con las características de ese hogar en la ciudad (Aón, López, Freaza, Giglio \& Cola, 2017b).

El análisis se desarrolla en cinco partes bien marcadas: la primera presenta y explica el marco teórico, junto con la estrategia metodológica desarrollada para este estudio. La segunda parte describe y caracteriza el caso, La Plata, en términos de configuración urbana, distribución de la población y caracterización de su sistema de salud, como base para explicar ciertos rasgos generales de la movilidad y accesibilidad a los hospitales públicos y privados. En la tercera parte se presentan los 16 hospitales relevados, con los respectivos resultados globales, para derivar en la cuarta sección al examen de dos casos específicos de los 16, con el fin de visibilizar ciertos rasgos característicos. El desarrollo y articulación de estas cuatro partes o secciones nos permiten argumentar y sustentar las reflexiones del último punto, sobre i) la desigualdad reflejada en el viaje por motivos de salud; y ii) la necesidad de desplegar políticas públicas para un desarrollo urbano más equilibrado y de acceso a la salud pública, como forma de completamiento de las políticas de equidad que, en materia de educación y salud, el Estado argentino viene sosteniendo desde hace muchas décadas.

$1 \longdiv { \text { Acorde al enfoque de los estudios del Observatorio de Movilidad de Gran La Plata, la incertidumbre } }$ de funcionamiento del sistema de transporte público colectivo automotor se refiere a la incerteza de su puntualidad o cumplimiento de las estaciones de parada definidas en los pliegos licitatorios y permisos de explotación que acuerdan las empresas de transporte público con los poderes ejecutivos municipales en las ciudades argentinas, mientras que la incertidumbre de inserción urbana se refiere a la incerteza de conseguir donde estacionar un automóvil particular en la ciudad. 


\section{El atractor de viaje como fuente de información}

En nuestro país se han aplicado históricamente estudios de detalle para la macroescala, basados en encuestas domiciliarias, de muy costosa implementación. Esta tradición ha reforzado la práctica de desarrollo de proyectos urbanos de movilidad sin estudios integrales previos y sin evaluación. Sin embargo, en la actualidad las metodologías de estudio de la movilidad han avanzado de manera acorde al desarrollo tecnológico y a la más reciente reflexión conceptual en torno al transporte y a la movilidad urbana y regional (Aón \& López, 2015).

Los mencionados estudios tradicionales y de costosa implementación para países como Argentina se estructuran a partir del concepto "generación de viajes", focalizando en uno de los dos componentes del binomio "producción-atracción" de viajes (Ortúzar \& Willumsen, 1994). En efecto, las encuestas domiciliarias de transporte procuran reconstruir la producción total de viajes de las ciudades desde los lugares de la producción y la generación de los mismos, que, de acuerdo con la teoría clásica, son los hogares. De esta manera, a partir de la información relevada mediante la encuesta de los hogares y sus miembros, se construyen las diferentes matrices de producción, atracción, generación, origen y destino de los viajes (figura 1).

FIGURA I | Esquema explicativo de las diferencias entre producción-atracción y origen-destino del viaje

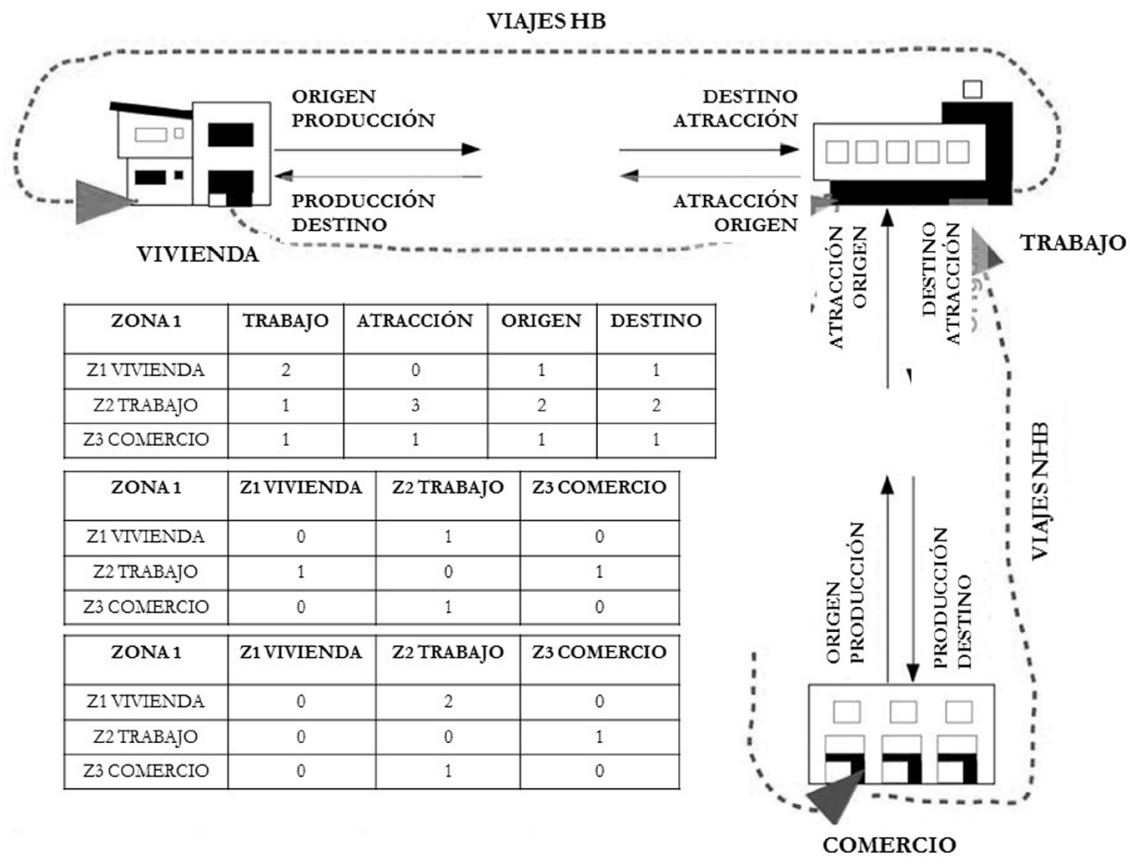

FUENTE AVANCES DE TESIS DOCTORAL, LAURA AÓN 
Basado en el planteo metodológico del Observatorio de Movilidad del Gran La Plata $^{2}$ (Aón, López, Freaza, Giglio \& Cola, 2017b), el presente estudio se focaliza en los atractores de viaje como fuente de información y análisis. De esta forma se desarrolla un camino inverso al tradicional, mucho más corto, construyendo información sobre la generación y producción de viajes a partir de encuestas relevadas en los atractores de viaje más importantes de la ciudad. Con tal fin, se toma como dato clave la referencia de ubicación de la vivienda y características del hogar de pertenencia de cada encuestado.

Entre las propuestas teóricas metodológicas de los modelos matemáticos de simulación de transporte, existen modelos algunos que se focalizan en destinos de viaje. Es el caso de los modelos gravitacionales y de los modelos Logit (Añez \& Pérez, 1999; Chasco, 2000; De la Barra,1999; Echeñique, 1975; Ortúzar \& Willumsen, 1994). Los primeros han sido utilizados para abordajes más agregados y escalas interurbanas; los segundos, para estudios centrados en los lugares de destino de viajes unitarios y con un mayor número de variables consideradas, relevadas mediante encuestas. En general, se focalizan en variables espaciales cuantitativas ligadas al diseño de infraestructuras y sistemas de transporte público. En cambio, nuestra estrategia metodológica de relevamiento, centrada en atractores, utiliza la teoría de la generación de viajes ligada a las características sociales y económicas del hogar, focalizando en el binomio producción-atracción de viajes en lugar de hacerlo en el binomio origendestino. Se constituyen en atractores de viaje actividades como las educativas, el empleo y la salud; es decir, actividades que implican importantes flujos de movilidad urbana.

Esta doble entrada permite el estudio de la movilidad desde los atractores de viaje como camino heurístico para acceder a la información de la generación de viajes de los hogares, incluyendo un conocimiento suplementario en la observación, análisis y estimación de las tasas de atracción de cada caso de estudio como marco de interpretación del levantamiento de encuestas a cada persona (Aón, López, Freaza, Giglio \& Cola, 2017b).

En la propuesta de investigación sobre atractores cobra relevancia estructural la identificación de la matriz productiva de cada ciudad. En el caso de la ciudad de La Plata, una de sus principales actividades productivas está ligada a la función como ciudad capital de la provincia de Buenos Aires. En materia de salud su rol es relevante, en primer lugar por la presencia de más de diez centros de salud especializados, y de alta complejidad, para nińos y adultos, ligados a la enseñanza universitaria de la medicina; y en segundo lugar, por la destacada oferta y capacidad de internación, que equivale a $63 \%$ del total de la región sanitaria que integra.

El estudio cuantitativo en los atractores de viajes de salud se realizó mediante encuestas estructuradas sobre la base de un formulario de preguntas que toma, por un lado, la definición del vínculo entre movilidad y acceso; y por otro, el nivel de facilidad o dificultad de una persona para acceder a un lugar. También recolecta

El Observatorio de Movilidad del Gran La Plata es una iniciativa del Grupo de investigación de transporte y movilidad de la línea 2 del Instituto de Investigaciones y Políticas del Ambiente Construido (IIPAC), Facultad de Arquitectura y Urbanismo (FAU), Universidad Nacional de La Plata (UNLP). Véase https://observatoriomovilidadlaplata.wordpress.com/\#content-wrapper 
información sobre el hogar y la vivienda, buscando construir información socioespacial y socioeconómica para abordar el análisis de atractores.

Es a partir de estas ideas que se construye la información del viaje específico del individuo y del hogar, dando cuenta de las siguientes condicionantes: i) espaciales ${ }^{3}$ (lugar de residencia); ii) materiales (nivel de ingreso); y iii) sociales (género, nivel de instrucción, problemas y motivaciones específicas del viaje al hospital).

Para la realización del estudio, el camino metodológico estratégico se diseñó en cuatro etapas: las dos primeras relacionadas a la organización y gestión previa en campo; y las dos restantes, a la producción, procesamiento de datos y análisis de información (figura 2).

La selección de casos fue realizada a partir de criterios acordes a las búsquedas de esta investigación: estudiar la criticidad del viaje a los centros de salud, analizar las diferencias de esa criticidad según grupos socioeconómico de la población, y reconocer particularidades relativas a la localización espacial de esos centros de salud en la ciudad.

Bajo estas lógicas, la selección final de los centros de salud (figura 3) llegó a ser representativa de los más importantes de la ciudad, en tamaño y capacidad de servicio que ofrecen, incluyendo públicos y privados, con localizaciones centrales, pericentrales, periurbanas y periféricas.

Para dimensionar la importancia del servicio de salud en cada caso, se consideraron factores como cantidad de consultas anuales, nivel de servicio, cantidad de trabajadores, pacientes y universitarios registrados diariamente y capacidad de internación, información obtenida en el Departamento de Estadísticas de Servicios de Salud y el Ministerio de Salud de la Provincia de Buenos Aires. Con tal información se elaboró una primera lista de establecimientos que categorizamos como atractores de alto impacto.

De la lista original de 26 establecimientos preseleccionados, el estudio abarcó solamente 16, por negativas administrativas de los propios centros de salud y falta de autorización de acceso al equipo de investigación para realizar el relevamiento.

La selección final concretada fue evaluada por el equipo de investigación, verificándose su representatividad respecto del sistema de salud platense según tamaño, tipo de gestión (pública/privada) y ubicaciones geográficas de los casos.

Durante la segunda etapa, el equipo se enfocó en la gestión y coordinación con el cuerpo directivo de cada centro de salud; y formatos, personal autorizado, fechas y horarios para la realización de las encuestas.

El operativo de campo se concretó en la tercera etapa, es decir, en el levantamiento de información a partir de la realización de las encuestas en cada centro de salud. Para tal fin, se trabajó con seis encuestadores reunidos en dos grupos en distinto turno: mańana y tarde. Cabe resaltar que todo el equipo de encuestadores tuvo una capacitación previa al trabajo de campo, en relación con el formato, los objetivos de la encuesta y el procesamiento de los datos. 
FIGURA 2 Etapas metodológicas

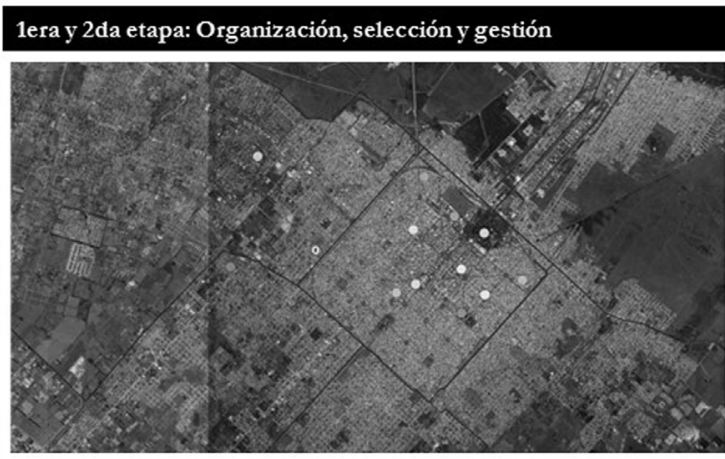

3ra y 4ta etapa: producción y procesamiento

6 encuestadores por día

4 semanas

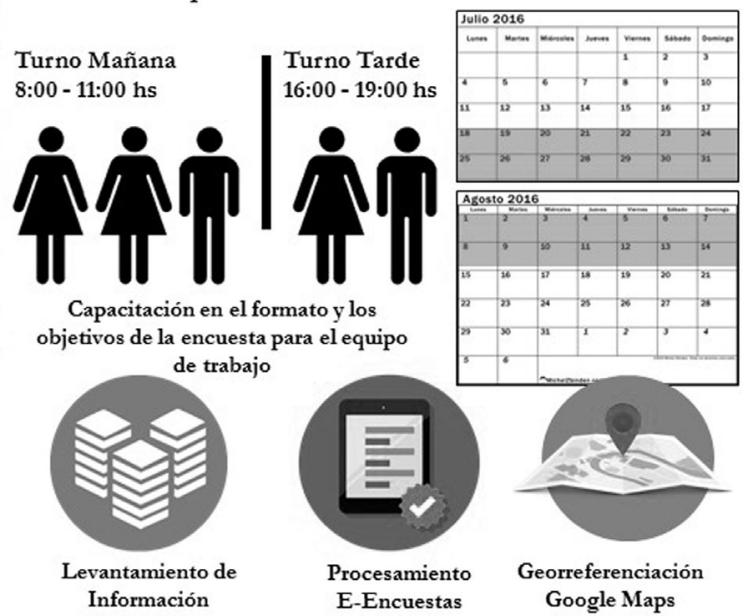

FUENTE OBSERVATORIO DE MOVILIDAD URBANA DEL GRAN LA PLATA, IIPAC CONICET UNLP. ELABORACIÓN GRÁFICA: MARÍA LUCIANA GIGLIO

Los destinatarios de la encuesta incluían pacientes, acompañantes y personal de cada centro de salud, incluyendo médicos, administrativos, enfermeros y personal de limpieza. La muestra o cantidad de encuestas se estimó según estadísticas y conforme a cada establecimiento. En total fueron realizadas 1.899 encuestas.

Para cada registro, correspondiente a todas las respuestas por parte de un solo encuestado, se asignó un número de identificación o ID, el cual permitía vincular la información alfanumérica producida por la encuesta con la localización espacial residencial de cada encuestado, en un sistema de información geográfica.

El formulario se estructuró en seis secciones: i) perfil del encuestado; ii) modo de transporte utilizado para llegar al hospital y problemas asociados a dicho modo; 
iii) relaciones de distancia y tiempo entre el centro de salud y su barrio y vivienda; iv) motivo específico por el que concurre a ese centro de salud; y vi) regularidad del viaje.

Los formularios destinados al personal se diferenciaban por registrar la labor que cada encuestado realizaba dentro del hospital, en lugar del motivo del viaje al centro de salud. A la vez, en estos formularios se dejaba espacio libre para detallar dificultades de viaje, conexiones con otros centros de salud, horas de entrada y salida y relación con frecuencias del transporte público.

La cuarta etapa se consignó al procesamiento de las encuestas y la vinculación georreferenciada, cargándose los datos diariamente en una plataforma web contratada, por medio de la cual se convertía en base para relacionar dichos datos a la georreferenciación de los lugares de residencia de los encuestados.

El mapeo se realizó por medio de Google Maps, instrumento compatible con el sistema de información geográfica. El procesamiento se realizó también por cada encuestador, y luego la vinculación y combinación de todos los datos estuvo a cargo de un coordinador.

Cabe aclarar que al gestionarse en la primera etapa información estadística de cada centro de salud, se calculó el tamaño de la muestra necesaria, para luego expandirla y estimar la demanda total de viajes por salud como punto de partida para la formulación de propuestas y medidas de transporte.

\section{Figura 3 | Sistema de Salud ciudad de La Plata}

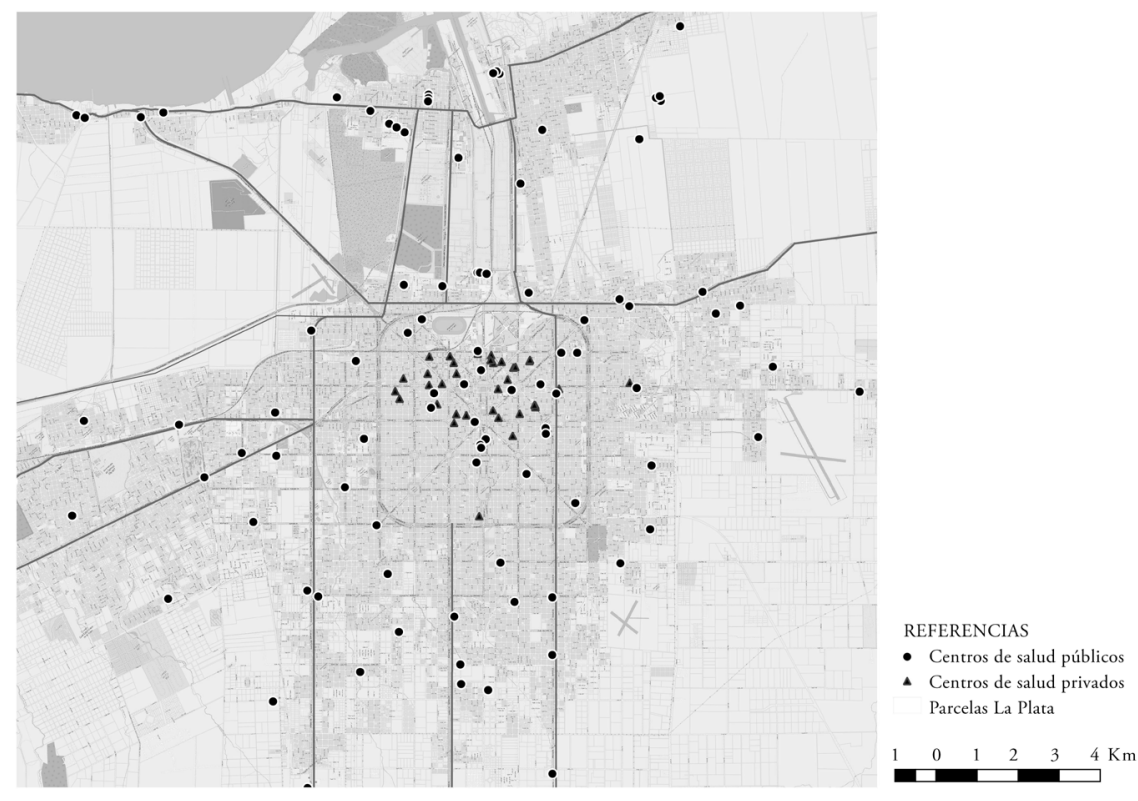

FUENTES MINISTERIO DE SALUD DE LA ARGENTINA 2OI 5, FLECLIBA, 20 I 8 Y OBSERVATORIO DE MOVILIDAD URBANA GRAN LA PLATA, 20I6. ELABORACIÓN GRÁFICA: MARÍA JULIETA LÓPEZ 


\section{Caracterización de La Plata y su sistema de salud}

La ciudad de La Plata (figura 4) es la capital de la provincia de Buenos Aires, está ubicada a $60 \mathrm{~km}$ de Capital Federal, y cuenta con aproximadamente 654.000 habitantes, según el Censo 2010. Este municipio se posiciona desde su fundación como nodo atractor y ciudad cabecera de la Microrregión del Gran La Plata, que incluye a los municipios vecinos portuarios de Berisso y Ensenada, ya que la actividad portuaria se conjuga con la localización de las autoridades gubernamentales provinciales, la administración municipal y la presencia de la Universidad Nacional de La Plata.

\section{FIGURA 4 La Plata, ubicación geográfica y configuración urbana}

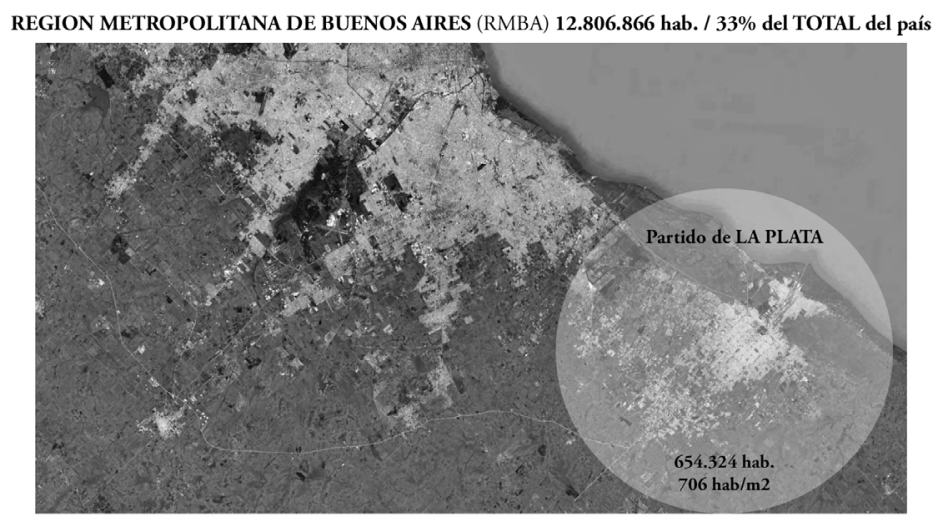

FUENTE GOOGLE EARTH

En cuanto al desarrollo urbano, la ciudad se fundó (1882) siguiendo en su concepción la integración del sistema de transporte: red ferroviaria, red de caminos y sistema de tranvía articulado, con el esquema de ciudad proyectado. Esta concepción se fue perdiendo con el transcurso del tiempo y en el contexto de la globalización de los mercados y del capital financiero, siguiendo las tendencias urbanas posindustriales, que dieron prioridad al desarrollo de equipamientos de consumo y urbanizaciones cerradas (López, 2015).

Actualmente se dan dos procesos simultáneos: la densificación del área central y la expansión de la periferia, en la que se pueden reconocer nuevas centralidades sobre corredores de crecimiento (figura 5), que se diferencian por su configuración urbana y perfil socioeconómico.

En cuanto al área central o casco urbano, se encuentra delimitado por el boulevard de Circunvalación, en el cual se definen tres barrios: Casco Norte, Oeste y Sur. La densificación y concentración de actividades se orienta por normativas ad hoc, y sigue las pautas del mercado inmobiliario y de los sectores privados. Según el Censo 2010, residen en esta área 166.271 habitantes (32\% del total); en ella, debido a la ausencia de planificación, es crítica la falta de adaptación de los servicios y equipamientos urbanos a los nuevos niveles de población. En este sentido, caos de tránsito, contaminación y accidentes son parte del escenario habitual para los ciudadanos. 
En cuanto a las cuatro zonas de la periferia, la norte está integrada por siete delegaciones: Tolosa, Ringuelet, M. B. Gonnet, City Bell, Villa Elisa, Hernández, Gorina. Esta zona es la que mayor consolidación presenta, con un tejido continuo residencial de tipo lineal y una población de 130.018 habitantes $(26 \%$ del total del partido), con un perfil socioeconómico medio y medio-alto. Su crecimiento se estructura en torno a tres vías regionales metropolitanas: el Camino Parque Centenario, el Camino General Belgrano y el Ferrocarril General Roca. Estas vías concentran la mayor parte del comercio y el tránsito interurbano liviano y público del Gran La Plata.

FIgURA 5 | La Plata proceso de crecimiento y distribución población

\section{La Plata: proceso de expansión sobre ejes viarios}
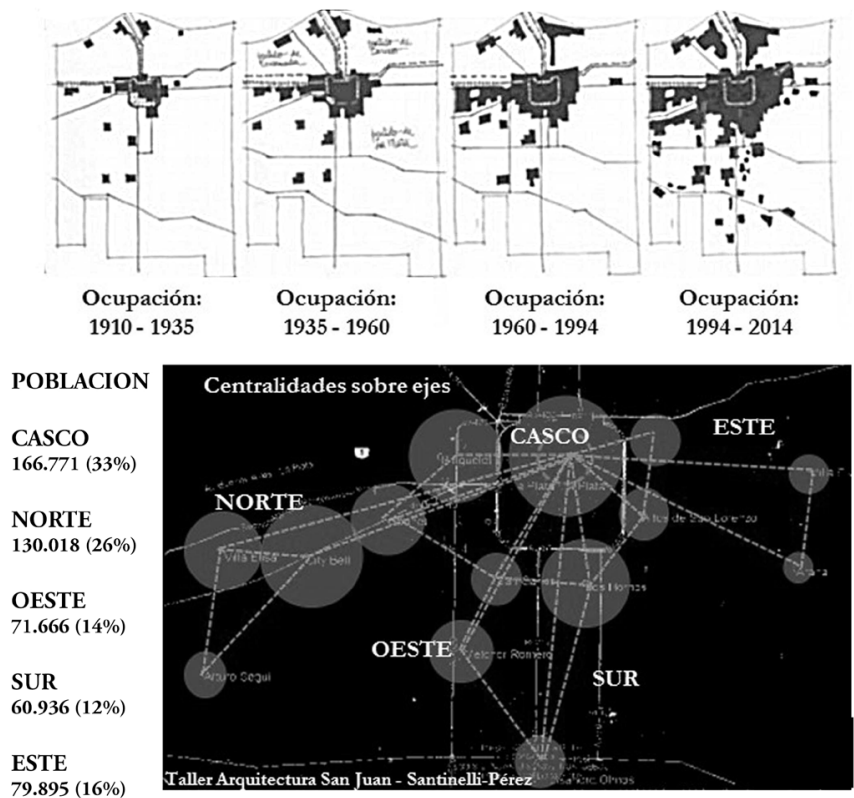

FUENTES DIRECCIÓN DE PLANEAMIENTO DE LA LA PLATA Y CATEDRA DE ARQUITECTURA SAN JUAN, SANTINELLI Y PEREZ, 2OI 8. ELABORACIÓN GRÁFICA: MARÍA JULIETA LÓPEZ

La zona oeste cuenta con una población de 71.666 habitantes (14\% del total del partido), caracterizada por un perfil socioeconómico medio-bajo; presenta un tejido continuo de tipo lineal y uso mixto, con presencia de centralidades secundarias.

Las zonas este y sur tienen un carácter eminentemente agropecuario. La primera, con 79.865 habitantes (16\% del total), un perfil social medio y medio-bajo similar a la zona oeste, pero con un tejido discontinuo y predominantemente residencial, sin presencia de centralidades marcadas. La zona sur es la menos poblada, con 60.936 habitantes (12\% del total del partido); se caracteriza por un perfil social medio y medio-bajo, y un tejido residencial y continuo con presencia de una fuerte centralidad, rodeado de amplias zonas de explotación agropecuaria. 
Con respecto al sistema de salud platense, al igual que en el resto del territorio de la República Argentina, la ciudad de La Plata conforma su servicio a partir del sistema público municipal y provincial (77 establecimientos), los prestadores privados (44 centros) y el sistema de obras sociales. Con la aprobación de la Ley 11.554 (1994), esta red sanitaria comenzó a formar parte de una de las doce regiones de la provincia de Buenos Aires. Así, La Plata, junto con 17 municipios, conforma la Región Sanitaria XI e intenta dar servicio a todos los habitantes de la zona.

En este contexto, el municipio se constituye en un nodo atractor muy importante no solo a nivel regional, sino también provincial y nacional, ya que recibe aproximadamente 2.126.666 millones de consultas anuales; es decir, un 40\% de consultas a nivel región. Esto se explica, en primer lugar, por la presencia de más de diez centros especializados, universitarios y de alta complejidad para niños y adultos; y en segundo lugar, por la destacada oferta y capacidad de internación, que equivale a $63 \%$ del total de la región y a un $8 \%$ de la provincia.

Si bien el nivel de prestación y servicio del sistema sanitario de La Plata es destacado, su distribución espacial en la ciudad (figura 6) está desbalanceada, al concentrase la mayor parte de los equipamientos en el área central. Ello implica que el patrón de accesibilidad dominante no está asociado a la proximidad de la vivienda, sino al tipo de gestión (pública o privada) o a la especialidad del centro de salud.

\section{FIgURA 6 | Centros de salud seleccionados}

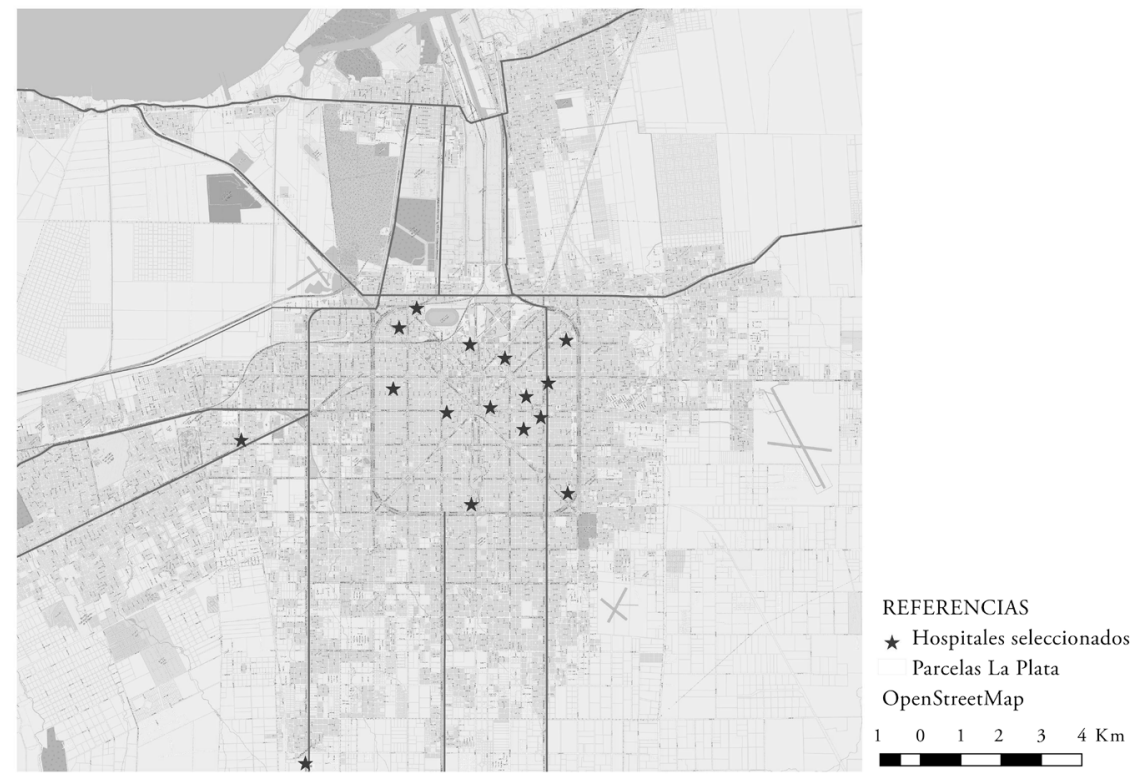

FUENTES MINISTERIO DE SALUD DE LA ARGENTINA 2OI5, FLECLIBA, 20 I 8 Y OBSERVATORIO DE MOVILIDAD URBANA GRAN LA PLATA, 2OI6. ELABORACIÓN GRÁFICA: MARÍA JULIETA LÓPEZ 


\section{Resultados de la encuesta de movilidad}

A partir de la georreferenciación y procesamiento de las 1.899 encuestas realizadas, se pueden reconocer ciertos problemas de viaje de los diferentes grupos de población encuestados, según su lugar de residencia y su perfil socioeconómico, aspectos que condicionan el viaje y el modo de transporte utilizado.

A continuación se exponen estos análisis sobre los resultados del estudio realizado en los 16 centros de salud, de donde surge la diferencia particular de casos según el tipo de gestión (pública o privada).

\section{Resultados generales relativos a los 16 centros de salud estudiados}

\section{Sobre la población encuestada}

Respecto de la población encuestada, más del $70 \%$ de las personas que viajan a hospitales son mujeres, y el viaje se distribuye en transporte público o auto de alquiler, según: i) condiciones socioeconómicas; o ii) grado de urgencia. Estos datos destacan la predominancia del rol de la mujer y la complejidad que este rol supone en la configuración de los viajes por motivos de salud.

Por otro lado, se registró una tendencia al "doble viaje", ya que la persona que necesita asistencia de salud viaja con un acompañante. Los resultados de las encuestan revelaron que, en un $60 \%$ de los casos, se trata de "viajes dobles", realizados por personas de entre 18 y 45 años. Esta tendencia supone una condicionante para la decisión del modo y tiempo de viaje.

\section{Sobre el viaje al hospital: modo y dificultades}

De los resultados generales, se registró un reparto modal bastante equilibrado entre el uso de automóviles particulares y buses. El 36,97\% del total de viajes registrados se realiza en automóvil particular, mientras que en el 36,89\% de los casos se resuelve en transporte público colectivo automotor. El 26\% restante de viajes se distribuye de manera heterogénea entre los demás modos de transporte, destacándose el viaje en remisse, con un 9,32\%; el viaje a pie, con un 6,85\%; y el viaje en taxi con un 3,43\%.

Este reparto modal total corresponde a todos los casos analizados, que incluyen patrones de viaje a hospitales públicos y privados, centrales y periféricos. Los patrones modales de viajes por salud se distribuyen de manera diferente en cada caso; especialmente, las diferencias radican en el tipo de gestión del centro de salud (pública o privada) y la relación que ello guarda con el perfil socioeconómico de la población que utiliza tales servicios. Así, en la mayoría de los casos es predominante el uso del transporte público para acceder a los centros de salud de gestión pública, mientras que tiende a predominar el uso del automóvil particular y de alquiler en el viaje a los centros de salud de gestión privada. La muestra tomada indica que más del $60 \%$ de los pacientes de hospitales públicos viaja en transporte público y un $20 \%$ en automóvil. En los centros de salud de gestión privada, estos valores se invierten.

Acorde con estos patrones, el registro de dificultades percibidas para realizar el viaje incluye, en primer lugar, los altos costos de las tarifas de transporte público. Este tema, ligado a la producción de "viajes dobles" por salud, los cuales claramente serán de ida y de vuelta, explica más profundamente el nivel de la dificultad percibida 
por los usuarios de esta modalidad de viaje. Los usuarios del automóvil particular no dieron cuenta de percibir mayores costos en el uso de este modo de transporte, aunque seńalaron como problema las dificultades para encontrar estacionamiento. En segundo lugar, se registra como problema percibido tanto el tiempo de espera para tomar el bus, como la distancia grande de caminata para acceder a las paradas del transporte público.

\section{Los problemas del transporte público según los usuarios}

Los problemas señalados por los usuarios de transporte público colectivo de pasajeros incluyeron, en forma particular: i) el tiempo de espera para tomar el transporte $(19,63 \%) ; y$ ii) las frecuencias del transporte público $(17,47 \%)$.

Podemos inferir que estos problemas están ligados a la forma no planificada de expansión de la ciudad y a la dificultad del sistema público de transporte para adaptarse y mejorar la oferta en las zonas de la periferia de baja densidad. A ello se suma que, en situaciones periféricas desprovistas de equipamiento urbano, el tiempo de espera es percibido de manera más crítica que en las áreas centrales, por el riesgo que supone la espera prolongada en lugares solitarios y deteriorados.

\section{Los problemas del uso del automóvil particular}

Es relevante analizar que los problemas señalados en el uso del automóvil particular van desde el mal estado de las calles $(22,27 \%)$ y la dificultad de encontrar estacionamiento $(21,48 \%)$, hasta los altos costos del combustible $(17,05 \%)$ y el estrés por conducir en contextos de alta congestión (11,82\%).

Los problemas percibidos están vinculados de manera directa al intenso uso del automóvil particular en la ciudad y a las tasas de motorización de las personas en la ciudad de La Plata, que hoy asciende a 1 automóvil cada 1,8 habitantes, lo cual supone unos 350.000 automóviles circulando.

En cuanto al deterioro de la infraestructura viaria, se trata de un problema que afecta a la ciudad en toda su extensión. En las áreas centrales, los problemas de la red viaria incluyen la falta de segregación para algunos modos, invasión de calles con estacionamientos medidos y ausencia de acompańamiento sistémico de semáforos para agilizar la circulación en el centro. En áreas periféricas, la red vial está dañada, sin banquinas, intransitable en circunstancias climáticas como las lluvias intensas. En ambas situaciones, la infraestructura vial afecta tanto al transporte privado como al público.

\section{Sobre los problemas del uso de motocicletas y bicicletas}

Los usuarios tanto de moto como de bicicleta tienen como principal preocupación el problema de riesgo de accidentes, seguido por el riesgo de robos.

El uso de la moto, por su tamaño, es un modo que permite "salvar el tiempo de viaje incrementado" que produce la alta congestión vehicular en el centro de la ciudad, en particular durante las prolongadas horas punta. Más allá de esta ventaja, sin embargo, la tasa de accidentes fatales de motociclistas en la ciudad es la más alta del país. Según datos de la Fundación Corazones Azules, todos los días muere un motociclista en la ciudad. 


\section{Sobre problemas de los peatones}

Acorde a la valoración de las infraestructuras de circulación para los usuarios de autos y buses, los peatones también declararon como principales problemas del viaje a pie el mal estado de las veredas $(34,61 \%)$. También perciben y declaran el riesgo de robos $(29,75 \%)$ y de accidentes (11\%).

Para los peatones significa un problema la cuestión del clima (13\%), y aunque el déficit en veredas y paradas aparece en las áreas centrales de la ciudad, claramente en áreas periféricas es mucho más crítico. Afecta tanto las paradas de buses como las veredas o calles, las cuales se vuelven extremadamente difíciles para la circulación en situaciones de intensas lluvias, hasta incluso impedir la espera o el viaje.

Recapitulando, podemos decir que a partir de este análisis integral de las encuestas realizadas a todos los centros de salud, se pueden reconocer cuatro aspectos particulares: i) la predominancia de mujeres en los viajes por salud; ii) el peso de los "viajes dobles"; iii) la relación proporcional de viajes en transporte público en los hospitales públicos y de viajes en auto en los privados; iv) la valoración y percepción de la calidad de las infraestructuras y riesgo de accidentes.

Estas cuatro cuestiones ayudan a explicar en mayor profundidad las condicionantes del viaje planteadas inicialmente como hipótesis, donde expresamos que tanto el nivel de ingreso de las personas, ligado a su disponibilidad o tenencia de automóvil particular, y el lugar de residencia, constituían aspectos explicativos de los problemas y desigualdades de acceso a la salud por parte de la población de la ciudad de La Plata.

\section{Resultados relativos a los dos casos seleccionados para el estudio}

Para entender mejor las diferencias seńaladas, a continuación se describe y muestran los resultados detallados de dos casos seleccionados: el Hospital Interzonal General de Agudos General José de San Martín, de gestión pública; y el Hospital Italiano, de gestión privada. Ambos son representativos de la muestra tomada y se diferencian en el tipo de gestión.

Estos dos centros se seleccionaron con el fin de mostrar los rasgos distintivos encontrados en relación con nuestras condicionantes analíticas de partida, que incluyen el nivel de ingreso de las personas, la tenencia o disponibilidad de automóvil y el lugar de la ciudad donde viven, y la forma en que tales condicionantes repercuten en la accesibilidad al servicio y en la complejidad del viaje.

\section{El hospital público:}

\section{Hospital Interzonal General de Agudos General José de San Martín}

El Hospital Interzonal General de Agudos General José de San Martín (figura 8) es un hospital universitario de referencia provincial, para la patología aguda y crónica de alta complejidad en adultos, con desarrollo en emergencias y trauma, trasplantes, oncología y servicios clínicos, quirúrgicos, de diagnóstico especializado, incluyendo salud mental. Además, es referencia regional en la atención perinatal de alta complejidad y en fertilización asistida. La asistencia que presta, y la cantidad de trabajadores, pacientes y universitarios que en él confluyen, lo posicionan como un atractor de Alto Impacto. 
Este centro de salud se ubica en el casco urbano al interior de una zona de escala barrial que no ha sufrido cambios importantes en cuanto a su morfología. Puntualmente, su ubicación sobre avenida 1, entre circunvalación y calle 69 , determina el excelente acceso al establecimiento. Según la muestra realizada, la razón por la cual se opta por su servicio se distribuye entre: i) las especialidades del hospital (33\%); ii) la derivación (26\%); iii) su cercanía (22\%); iv) presencia de médico de confianza y atención gratuita $(18 \%)$.

El registro de género evidencia que más del $76 \%$ de las personas que viajan a este hospital son mujeres. Más de la mitad de los viajes (52\%) se realizan en compañía de otra persona. El perfil laboral se manifiesta muy variable entre los encuestados.

A partir de la georreferenciación espacial de los pacientes, se ve una fuerte demanda de las zonas este, oeste y sur de La Plata, caracterizadas por su perfil social económico medio-bajo. Como consecuencia, en los viajes hacia y desde el hospital predomina el uso del transporte público automotor $(54,17 \%)$, que se posiciona por sobre el automóvil particular (20\%). El 26\% restante se distribuye entre los demás modos.

\section{FIGURA 7 Hospital San Martín}

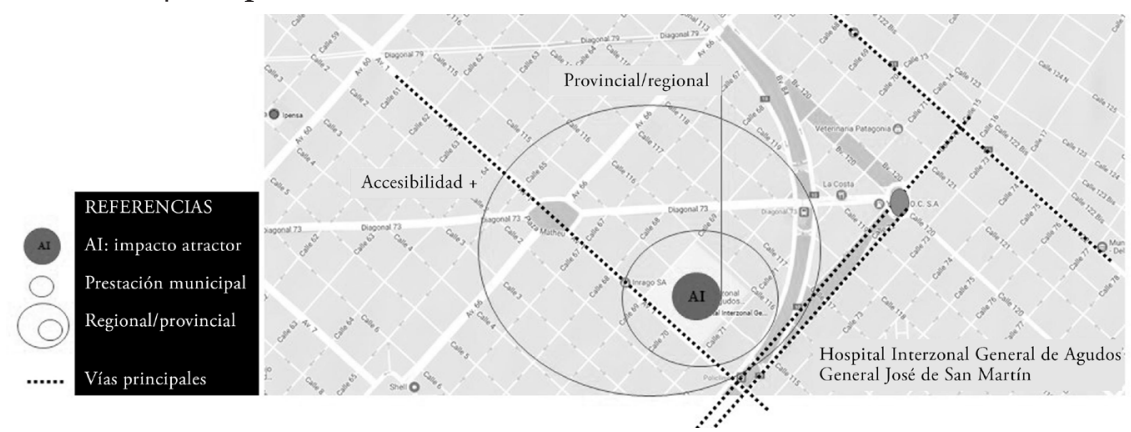

FUENTES ELABORACIÓN PROPIA

En cuanto a las dificultades para realizar el viaje, destacan el tiempo de espera, la caminata para tomar el transporte público y, por sobre todo, el costo de la tarifa. La falta de coordinación de las frecuencias hace que colapsen las colas y la subida a los diferentes coches.

En relación con las líneas mayormente utilizadas (Este y Oeste), la principal queja se vincula a la incertidumbre de llegada del transporte y a las bajas opciones con que se cuenta en caso de perder un autobús, caso en que se deben hacer combinaciones que claramente suponen más costos y tiempos para cada usuario. Esta molestia se expresa fundamentalmente en relación con una de las líneas directas, la más acusada de no frenar, frente a la urgencia de combinación (doble pasaje).

\section{El hospital privado: Hospital Italiano}

El Hospital Italiano en La Plata (figura 7) es una institución privada de mediana complejidad y de referencia provincial. Surgió como centro de asistencia para los primeros pobladores de la ciudad en la zona de la delegación de Los Hornos. Sus 
especialidades en cardiología y otras áreas lo han consolidado como un referente en salud a lo largo de los años.

La asistencia que presta, y la cantidad de trabajadores, pacientes y universitarios con que cuenta, lo posicionan como un atractor de alto impacto. Al igual que el caso anterior, se encuentra ubicado en el casco urbano, en una zona que viene sufriendo fuertes transformaciones por procesos constantes de densificación. Su proximidad a los principales accesos a la ciudad, la avenida 44 y circunvalación 31, también permite una accesibilidad óptima al establecimiento.

De la muestra realizada en este centro de salud, la razón por la cual lo eligen los encuestados se distribuye entre: i) por costumbre o tradición familiar; ii) porque atiende un médico de confianza; iii) por cercanía; iv) porque fueron derivados al mismo por los servicios de alguna Aseguradora de Riesgos del Trabajo (ART).

Con respecto al perfil de género de los encuestados que viajaron a dicho hospital, hay predominancia de mujeres (64\%). Por tratarse de un hospital de atención general, en más de la mitad de los casos (51\%) los encuestados realizaron el viaje al hospital solos, un $20 \%$ lo hizo en compañía de un adulto, y un 15\%, llevando a un adulto.

\section{FIGURA 8 | Ubicación Hospital Italiano}

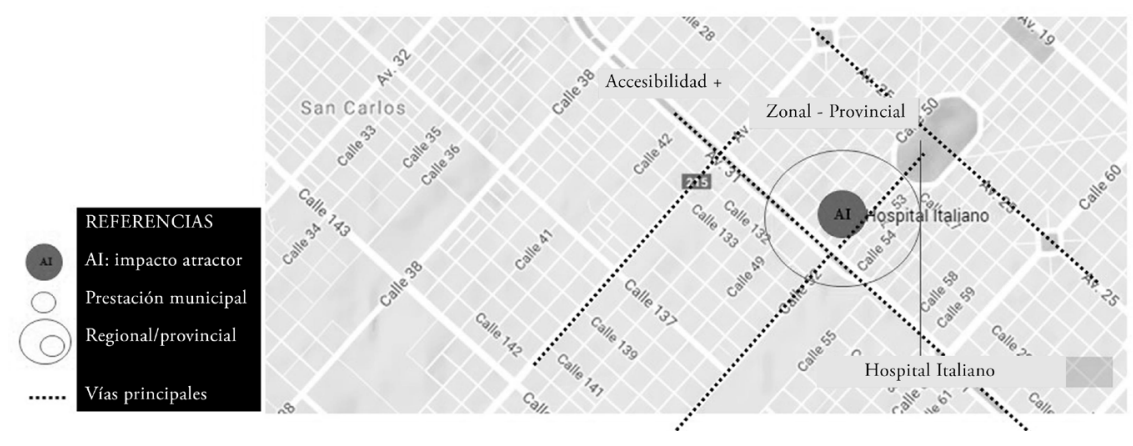

FUENTES ELABORACIÓN PROPIA

El perfil laboral de los encuestados en el hospital es variado; predominan los empleados del sector privado (28\%) seguidos por otro tipo de empleo $(27 \%)$ y por los empleados del sector público (20\%).

A partir de la georreferenciación de la vivienda de los pacientes, se evidencia una predominancia de pacientes residentes en el casco urbano y en las periferias sur y oeste. Se trata de los barrios más cercanos al Hospital Italiano y, para los casos de las periferias sur y oeste, son áreas con frecuencias de servicios de transporte público (Líneas Oeste, Sur y 506) altas y relativamente directas, con paradas en el Hospital Italiano (figura 9).

Para llegar al hospital, en este caso predomina el uso del automóvil particular, con el cual se realiza el $56,17 \%$ de los viajes, mientras que el transporte público 
colectivo automotor resuelve apenas el $17,28 \%$ de los casos, seguido por el modo remisse, con 13,58\%. El 13\% restante se distribuye entre viajes a pie, en moto y en bicicleta.

Entre las dificultades declaradas por los encuestados, destaca por sobre todo la relacionada con encontrar estacionamiento, así como los costos del transporte público y del combustible. Con respecto a la oferta de transporte público disponible en torno al hospital en referencia, se observa un déficit en el vínculo con los sectores norte y este de la ciudad, priorizándose un eje vertical de Berisso-Ensenada, hasta el área oeste del partido (figura 9).

Las líneas más utilizadas, como la 307 y la Oeste, ofrecen una adecuada cobertura y accesibilidad al Hospital Italiano, aunque presentan déficit de frecuencia en el servicio, lo cual desestimula su uso por parte del usuario. Esto acarrea un mayor uso del auto particular y, en consecuencia, dificultades en el momento de encontrar un estacionamiento próximo al establecimiento, que se localiza en una zona residencial típica de la ciudad.

Comparando los dos casos seleccionados (figura 9) se comprueba la estrecha relación entre el tipo de gestión de los centros de salud, los modos predominantes de transporte utilizados en los barrios donde habitan los pacientes, y sus perfiles socioeconómicos. El Hospital San Martín da servicio a poblaciones residentes principalmente de las zonas este-oeste y sur de La Plata, que se caracterizan por un perfil socioeconómico medio y medio-bajo, donde predomina el viaje en transporte público. Por su parte, el Hospital Italiano da principalmente servicio a población que reside en las zonas de mejor situación socioeconómica, la cual viaja predominantemente en autos particulares o de alquiler.

Por otra parte, las distancias entre residencia y centros de salud suponen, en la mayoría de los casos, la necesidad de un modo motorizado. En cuanto al reparto modal de viajes, hemos señalado ya que el auto y el transporte público son los modos de transporte más utilizados, con predominancias dispares para cada caso. En este sentido, la desigualdad de oportunidades se agrava por las distancias que los distintos grupos de población deben recorrer.

Es preciso señalar que, en relación con un mismo centro de salud, se realizan viajes de personas con diferente nivel de ingreso y distinta modalidad de viaje; y ello, de una parte, por la cantidad de población de sectores populares que en años recientes comenzó a tener acceso a prestaciones sociales de salud; pero, por otra, debido a los diferentes tipos de empleo involucrados en cada hospital. En este sentido, las autoridades de los hospitales entrevistadas reconocían que el personal de seguridad y limpieza predominantemente accedía a su trabajo cotidiano en transporte público, mientras que la situación del personal de enfermería no es tan homogénea. Este último grupo, que constituye un sostén estructural del funcionamiento de los hospitales, tiene una jornada laboral de ocho horas en los de gestión privada, mientras que en los hospitales de gestión pública su jornada es de seis horas de trabajo. Estos movimientos de grupos de enfermeros que salen y entran en simultáneo para reemplazarse y sostener de manera permanente los servicios de internación, constituyen un tema pendiente de considerar en el diseńo de los recorridos del sistema de transporte público colectivo automotor. 
FIGURA 9 | Comparación de cada caso. 1) Ubicación encuestados. 2) Reparto modal. 3) Oferta de transporte público. 4) Dificultades de viaje
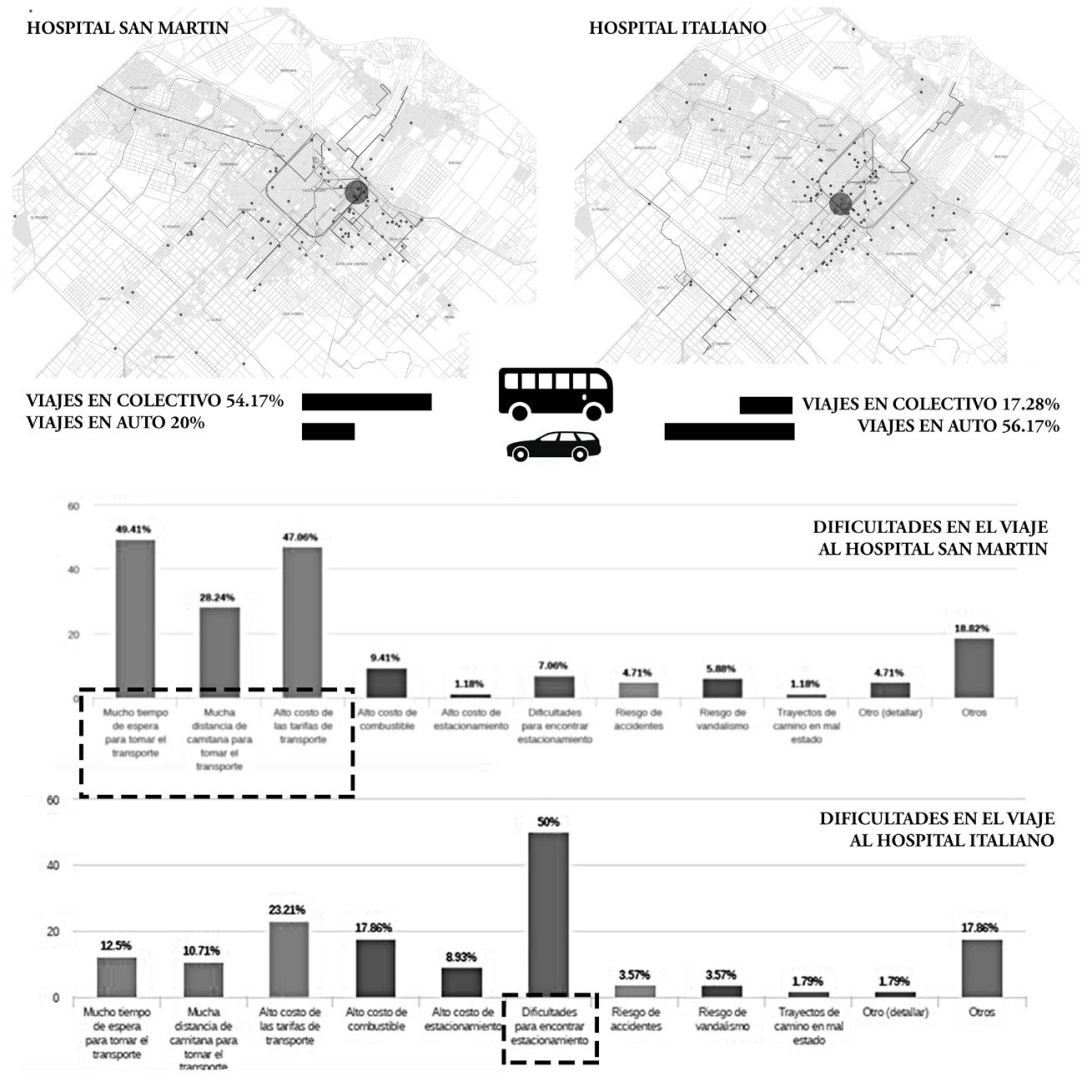

FUENTE ELABORACIÓN PROPIA

En líneas generales, el diseño del sistema de transporte público colectivo automotor presenta deficiencias de acceso en los dos casos analizados, lo que, sumado a las dificultades de frecuencias, induce a que las personas desplieguen complejas estrategias para acceder al hospital mediante transporte público.

De acuerdo con relevamientos espaciales y relatos escritos de las experiencias realizadas, los cuales fueron elaborados por los coordinadores del trabajo de campo en hospitales, las situaciones propias del entorno urbano en cada caso involucran congestión vehicular y de buses. Estas particulares condiciones urbanas del entorno de los hospitales, cualifican y califican negativamente la alta oferta de líneas de transporte público que llegan a ellos. La evaluación empírica realizada para las situaciones de espera en paradas de las diferentes líneas evidencia un colapso en la relación espacio-personas, y se valida en la apreciación de los encuestados. 


\section{Reflexiones}

De acuerdo con los análisis y relatos expresados en este trabajo, podemos concluir de manera general que el viaje al hospital se constituye en barrera en la medida en que se dispone de menos recursos económicos para resolverlo o en la medida en que mayor sea la distancia que se requiere recorrer para concretarlo.

Los resultados obtenidos de la muestra general dan cuenta de las diferentes situaciones que se presentan en relación con la movilidad por salud en la ciudad de La Plata, entre las cuales han quedado en evidencia las grandes desigualdades en el viaje al hospital y, por lo tanto, la desigualdad en el acceso a la salud.

Asimismo, el trabajo consolida argumentos para diseñar políticas públicas que consideren en conjunto el desarrollo urbano y el acceso a la salud como forma de completamiento de las políticas de equidad, focalizando en promover una mayor inclusión a la salud para todos los sectores de población. Algunos resultados específicos permiten reconocer aspectos clave que pueden incidir en cambios en las políticas de transporte y de salud, orientados a mitigar los efectos de la desigualdad e inequidad en el acceso a los servicios urbanos. En este sentido, podemos sintetizar ideas que pueden constituirse en insumos para los actores decisores:

En primer lugar, se destaca la componente relativa a género detectada en la encuesta, es decir, la predominancia de la presencia femenina en los espacios de la salud. Este aspecto en particular merece especial atención en materia de seguridad física de las mujeres, tanto en los tiempos de espera como durante el viaje, al igual que en los entornos de los centros de salud o zonas barriales, completando para ello su materialidad física de infraestructuras y luminarias, de espacios cómodos de espera.

La segunda componente destacada en este trabajo es la necesidad de incluir los temas de edad y condición física debilitada de los pacientes, como objeto de políticas públicas relacionadas con la renovación de infraestructura y equipamiento urbano. Nos referimos a problemas típicos en el ámbito de la salud —como la movilidad restringida habitual en los pacientes_- que requieren componentes específicas en el diseño de veredas, rampas, accesos y circulaciones, para garantizar el acceso a los servicios que ofrece el hospital. Estos temas incluyen no solamente aspectos de la accesibilidad espacial, como estacionamientos y rampas, para las personas con dificultades de movilidad; además, remiten a la posibilidad de tener acceso a nivel en el transporte público masivo.

El tercer aspecto se relaciona con las principales problemáticas declaradas por los usuarios con respecto a los modos de transporte que predominantemente utilizan, tanto en el caso de los usuarios de automóvil particular y de alquiler, como en el de los usuarios de buses. Al respecto, un tema que debe ser objeto de política pública es el relativo al sistema de transporte público colectivo automotor, su diseño de recorridos y frecuencias, y las estrategias del municipio y empresarios para sortear las dificultades de la congestión de tránsito cotidiana que entorpece la circulación de colectivos y retrasa su llegada a cada destino. En cuanto a los usuarios del automóvil particular, hemos señalado que sus principales preocupaciones se relacionan con las dificultades que tienen al llegar al hospital para encontrar estacionamiento. 
Estas dos problemáticas muestran aspectos esenciales de dos sectores socioeconómicos diferenciados, uno con vehículo propio y el otro cautivo del colectivo urbano. Ambos grupos tienen dificultades para materializar su acceso al hospital y esperan la solución a este problema por parte del Estado. Ante esta demanda, la posibilidad de crear estacionamientos es una política dirigida a los sectores que poseen un medio de transporte privado incentivando su uso, mientras que la promoción de mejoras en el transporte colectivo a partir de plantear nuevos recorridos, mejores frecuencias y garantizar la calidad del viaje, constituiría una medida dirigida a toda la sociedad en su conjunto.

La cuarta componente clave remite a la relevancia de trabajar en una oferta de servicios de salud diversificada, procurando garantizar una distribución espacial acorde a los barrios y la población del partido. Hemos señalado en los análisis de resultados que los motivos por los que concurren los pacientes a los hospitales muchas veces están relacionados con sus necesidades físicas directamente vinculadas a las afecciones que buscan resolver, o con categorías de prestación de servicios, o la garantía de calidad de los mismos.

A partir de todo lo expuesto y para sintetizar los aportes de esta comunicación, se busca concientizar a todos los actores involucrados sobre la necesidad de asumir las diferencias y las complejidades de los viajes de salud en el diseño de políticas que promuevan el uso de modos de transporte públicos masivos, de manera tal que el viaje no constituya una barrera más en el acceso a los servicios urbanos, como los vinculados a la salud.

El trabajo validó la metodología planteada a partir de las reflexiones conceptuales, y sus resultados muestran las posibilidades que ofrece el trabajo mediante plataformas digitales y con los sistemas de georreferenciación utilizados.

Asimismo, da cuenta ampliamente de que indagar y estudiar a partir del concepto "generación de viajes", focalizando en la atracción de viajes, implica una ventaja metodológica, porque permite investigar la experiencia individual combinada en el atractor. Por esta vía es posible acceder a temas problemáticos que pueden quedar fuera del relato de los encuestados, pero que puede registrar el observador investigador como contexto de significado más amplio, y a la vez complejo, de los relatos de individuales.

En este sentido, desde lo metodológico consideramos oportuno impulsar la implementación de metodologías expeditivas, y de bajo coste y alta confiabilidad, para estudios de movilidad urbana en contextos como Argentina, donde se destinan presupuestos acotados para diagnósticos y planificación. 


\section{Referencias bibliográficas}

Abba, A., Goicoechea, M. E., Furlong, S. \& Susini, S. (2015). El mapa social de la Región Metropolitana de Buenos Aires en 2010. Una caracterización de la estructura socioterritorial y una mirada a las transformaciones recientes a partir de los mapas sociales. En S. Vidal-Koppmann (comp.), Metrópolis en mutación (pp. 17-55). Buenos Aires: Café de las Ciudades.

Abba, A., Kullock, D., Novick, A., Pierro, N. \& Schweitzer, M. (2011). Horacio Torres y los mapas sociales: La construcción teórica del caso Buenos Aires. Buenos Aires: Cuentahilos. https://bit.ly/2sDdx $5 \mathrm{~N}$

Aón, L. \& López, J. (2015). Aplicación de nuevas tecnologías y recuperación de viejas prácticas de gestión de información para la planificación y gestión de la movilidad y la ciudad. Primer Congreso Argentino de Transporte 2015 (pp. 79-89). Ciudad Autónoma de Buenos Aires: Eudeba / Programa Interdisciplinario de la Universidad de Buenos Aires de Transporte (PIUBAT) / Universidad de Buenos Aires. https://bit.ly/2HnISOs

Aón, L., Giglio, L. \& Cola, C. (2017). Patrones modales de movilidad y desarrollo urbano no planificado en la ciudad de La Plata. Revista Transporte y Territorio, (17), 117-144. http://revistascientificas.filo.uba.ar/index.php/rtt/article/view/3870

Aón, L., López, J., Freaza, N., Giglio, L. \& Cola, C. (2017a). Observatorio de Movilidad Urbana Gran La Plata. Tomo 2: Atractores de viaje por Salud. La Plata: Instituto de Investigación y Políticas del Ambiente Construido, Facultad de Arquitectura y Urbanismo, Universidad Nacional de La Plata. https://drive.google.com/file/ d/1K7FGeJtBCi-qWoadHfzVx41b-6fUkz7m/view

Aón, L., López, J., Freaza, N., Giglio, L. \& Cola, C. (2017b). Patrones de movilidad a nivel de hogar en el Gran La Plata. La Plata: Universidad Nacional de La Plata.

Bourdieu, P. (2000). Las estructuras sociales de la economía. Buenos Aires: Manantial.

Chasco, P. (2000). Modelos de gravitación comercial: una aplicación al anuario comercial de España. Madrid: Universidad Autónoma de Madrid. https://www.uam.es/otroscentros/klein/ docjor/pchasco.pdf

Chaves, M. (2005). Construyendo ciudadanía: Tres acontecimientos para leer juventudes, prácticas culturales y políticas del estado. IV Jornadas de Sociología: La Argentina de la crisis: Desigualdad social, movimientos sociales, politica e instituciones. La Plata: Universidad Nacional La Plata. http://www.memoria.fahce.unlp.edu.ar/trab_eventos/ ev.6584/ev.6584.pdf

Chaves, M. (2008). Cuerpo y cultura: tres escenarios, muchos actores, un territorio final. Jornadas de Cuerpo y Cultura de la Universidad Nacional La Plata, 15 al 17 de mayo de 2008. La Plata, Argentina.

De la Barra, T. (1989). Integrated land use and transport modelling. Cambridge, uK: Cambridge University Press.

De la Barra, T., Añez, J. \& Pérez, B. (1999) Asignación Probabilística Multimodal. URBANA: Revista Eletrônica do Centro Interdisciplinar de Estudos sobre a Cidade, 4(25), 67-84. http://190.169.30.98/ojs/index.php/rev_urb/article/view/6625

Echenique, M. (comp.). (1975). Modelos matemáticos de la estructura espacial urbana: aplicaciones en América Latina. Buenos Aires: Ediciones siAP. 
Echenique, M. (1995). Entender la ciudad. EURE, 21(64), 9-23. https://www.eure.cl/index. $\mathrm{php/eure/article/view/1146}$

Figueroa, O. (2005). Transporte urbano y globalización. Políticas y efectos en América Latina. EURE, 31(94), 41-53. http://www.eure.cl/index.php/eure/article/view/1337

Gutiérrez, A. (2009). Movilidad y acceso: embarazo y salud pública en la periferia de Buenos Aires. XV CLATPU: Congresos Latinoamericanos de Transporte Público y Urbano. Buenos Aires. http://www.filo.uba.ar/contenidos/investigacion/institutos/geo/ptt/ GutierrezClatpu09a.pdf

Jirón Martínez, P. (2009). Prácticas de movilidad cotidiana urbana: un análisis para revelar desigualdades en la ciudad. En M. Tironi \& F. Pérez (eds.), scL/Espacios, prácticas y cultura urbana (pp. 176-189). Santiago: Ediciones Arq (Escuela de Arquitectura, P. Universidad Católica de Chile), Serie Teoría y Obra, Vol. 9. Versión escaneada en https://bit.ly/2swOyASy

Jirón Martínez, P. \& Fadda, G. (1999). The process of formulating quality of life indicators using a gender perspective. The need for "gendered" indicators in urban policy, programme and project analysis. Paper presented at Mainstreaming Gender in Policy and Planning South - North Experience, 28 June-1 July 1999. London, uk.

Jirón Martínez, P., Lange, C \& Bertrand, M. (2010). Exclusión y desigualdad espacial: Retrato desde la movilidad cotidiana. Revista INVI, 25(68), 15-57. http://dx.doi.org/10.4067/ S0718-83582010000100002

Landon, P. (2013). Movilidad cotidiana y exclusión social: anverso y reverso de la instalación de la autopista acceso sur en la periferia pobre de la metrópolis de Santiago de Chile. Encuentro Iberoamericano de Movilidad Urbana Sostenible (EIMUS). http:// www.ciudadenmovimiento.org/wp-content/uploads/2014/03/3er-Premio-PauletteLandon.pdf

López, J. (2015). Red viaria, ciudad y paisaje. Aproximación teórica metodológica para su diseño. Tesis doctoral. Facultad de Arquitectura y Urbanismo, Universidad Nacional de La Plata, Argentina http://sedici.unlp.edu.ar/handle/10915/63160

Losano, G. (2011). La industria en la Región Metropolitana de Buenos Aires: Los factores generadores de ventajas locacionales. En L. Adriani, M. Papalardo, P. Pintos \& M. Suárez, Actores, estrategias y territorio: El Gran La Plata: De la crisis de la convertibilidad al crecimiento económico (pp. 107-172). La Plata: Facultad de Humanidades y Ciencias de la Educación, Universidad Nacional de La Plata.

Olivera, H., Aón, L. \& Ravella, O. (2003). Evaluación de consumo y emisiones en dos propuestas de transporte Gran La Plata. Revista Averma. Avances en Energías Renovables y Medio Ambiente, 7(1), 01.23-01-28. https://www.mendoza-conicet.gob.ar/asades/ modulos/averma/trabajos/2003/2003-t001-a005.pdf

Ortúzar, J. (2000). Modelos de demanda de transporte. $2^{\mathrm{a}}$ ed. México, DF.: Alfaomega.

Ortúzar, J. de Dois \& Willumsen, L. G. (1994). Modeling Transport. $2^{\text {nd }}$ ed. Nueva York: John Wiley and Sons.

Ravella, O. (1994). Encuestas de Origeny Destino de Viajes. Informe Final. La Plata: Municipalidad de La Plata / Instituto de Estudios del Hábitat (IDEHAB), Facultad de Arquitectura y Urbanismo, Universidad Nacional de La Plata. 
Ravella, O. (2000). Modelos de evaluación de transporte en sistemas urbanos cerrados. (Informe final proyecto de Investigación. Consejo Nacional de Investigaciones Científicas y Técnicas (CONICET). La Plata: Universidad Nacional de La Plata, Argentina.

Segura, R. (2006). Segregación residencial, fronteras urbanas y movilidad territorial. Un acercamiento etnográfico. Cuadernos del Instituto de Desarrollo Económico y Social (IDES), n. ${ }^{\circ} 9$ (Buenos Aires). https://sociologia1unpsjb.files.wordpress.com/2008/03/ segura-segregacion-residencial.pdf

Segura, R. (2012). La trama relacional de la periferia de la ciudad de la plata. La figuración "establecidos-outsiders" revisitada. Publicar en Antropología y Ciencias Sociales, (10), 85-106 http://ppct.caicyt.gov.ar/index.php/publicar/article/view/1200

Vignoli, J. (2008). Movilidad cotidiana, desigualdad social y segregación residencial en cuatro metrópolis de América Latina. EURE, 34(103), 49-71. http://dx.doi.org/10.4067/ S0250-71612008000300003

Zucchini, E. (2015). Género y transporte: análisis de la movilidad del cuidado como punto de partida para construir una base de conocimiento más amplia de los patrones de movilidad. El caso de Madrid. Tesis doctoral. Escuela Técnica Superior de Arquitectura, Universidad Politécnica de Madrid. https://bit.ly/2sIThiw 